\title{
Stain capacity of three fungi on two fast-growing wood
}

\author{
Taize Song ${ }^{1} \cdot$ Fangchao Cheng $^{1} \cdot$ Jianping Sun ${ }^{1}$
}

Received: 26 September 2019 / Accepted: 25 October 2019 / Published online: 4 March 2020

(C) The Author(s) 2020

\begin{abstract}
We investigated the stain of fast-growing wood (Cunninghamia lanceolate, CL; Paulownia, PT) inoculated with three fungi (Arthrinium phaeospermum, AP; Vibrio anguillarum, VA; Aspergillacea, $A S$ ) to explore the new wood dyeing ways and the better combination of wood and fungi for dyeing. Only $A P$ could dye on $C L$ and $P T$. Especially for $C L$, its percentage of internal spalting, percentage of external spalting and dyeing depth were the highest (48\%, 15\% and $5.06 \mathrm{~mm}$, respectively). Surprisingly, the bigger weight loss occurs on $P T$. The results showed that the dyeing effect of $A P$ dyeing $C L$ was the best, and the wood color change was obviously (Orange to dark red). $A P$ could produce more pigments than the other two fungi $(V A ; A S)$, $C L$ was more suitable for fungus staining than $P T$, indicating that $A P$ could offered a new potential market and a chance for areas to earning higher income for $C L$. This research paves the way for improving color change was obviously (Orange to dark red). AP could produce more pigments than the other two fungi $(V A ; A S), C L$ was more suitable for fungus staining than $P T$, indicating that $A P$ could offer a new potential market and a chance for areas to earn higher income for $C L$.
\end{abstract}

Project funding: This research was supported by the Fundamental Research Funds for the National Natural Science Foundation of China (Project 31660174), and the InnovationDriven Project Funds of Guangxi (AA17204087-16).

The online version is available at http://www.springerlink.com.

Corresponding editor: Yu Lei.

Jianping Sun

jpsun@gxu.edu.cn

1 School of Resources, Environment and Materials, Guangxi University, Nanning 530004, People's Republic of China
Keywords Spalted wood C Cunninghamia lanceolata . Paulownia $\cdot$ Arthrinium phaeospermum $\cdot$ Vibrio anguillarum $\cdot$ Aspergillacea

\section{Introduction}

The demand of environmentally friendly wood dyestuff has been higher year by year owing to the economic development and the improvement of living standards, especially the people's awareness of environmental protection has been constantly enhanced. Therefore, many scientists have paid their attention to the researches on environmentally friendly wood dyestuff all over the world. Among these wood dyestuff, direct dyes, acid dyes and VAT dyes are usually employed to dye wood. However, these chemical dyes can cause serious air pollution, limiting the application of dyestuff in living space. Therefore, the pigment of environmentally friendly has great potential in the future. Among them, fungus pigment is regarded as a perfect wood dyestuff due to its environmental friendliness, which has attracted extensive research interest.

There is a long history of fungus pigment as a wood dye (Blanchette and Biggs 1992). Until now, fungus pigments have been used as a special dye to increase the value-added of wood, which depends on the colonization and stain wood ability of fungi (Robinson et al. 2007a, b; Robinson and Laks 2010a, b, c). How to approach the idealized technology of spalted wood is the goal of researchers in recent years. Therefore, exploring effective pigmented fungi and wood is to find the optimal environment of pigmented fungi.

Spalting is mainly divided into three parts: the stain fungi producing the stain substance, the bleaching formed by the white rot fungus, and the zone lines formed by the fungal interaction between the funguses (Robinson 2012). 
The dyeing and the zone lines are widely used in wood dyeing. In terms of fungal staining, Scytalidium cuboideum and Scytalidium ganodermophthorum are relatively effective fungus, which can produce red pigment and yellow pigment, respectively (Vega Gutierrez 2016). When spalted fungi is inoculated on wood, it can obtain nutrition in wood rays or other internal tissues of wood, thus dyeing wood. However, spalted fungi have white rot ability for wood (Liese 1970; Worrall et al. 1997; Richter and Glaeser 2015). Existing studies have shown that the longer the incubation time of dyeing fungi is, the better the dyeing effect of wood is, but the higher the risk of white rot of wood is. Therefore, in practical application, fungal pigments can be extracted by organic solvents such as DCM and applied directly to wood dyeing, or dissolved in flaxseed oil after extraction by organic solvents and then dyeing wood, which can solve the problem of white rot of wood and shorten the whole dyeing time (Robinson et al. 2014a, b; Robinson et al. 2014a, b; Hinsch et al. 2015; Weber et al. 2015; Agurto et al. 2017; Robinson et al. 2017; Hinsch and Robinson 2018). In terms of zone lines, the researchers found that Bjerkandera adustal Trametes versicolor and Polyporus brumalis/Trametes versicolor combinations, as well as X. polymorphism, Coriolus versicolor, etc. could produce stable zone lines (Robinson et al. 2010; Robinson et al. 2011). There are many reasons for the formation of zone lines, but generally they are all caused by changes in the growth environment, which are "Guard" and "Death" of hyphae (Mallett and Hiratsuka 1986; Cease et al. 1989).

In addition, fungi have different preferences for wood because of different functional groups and nutrients received from wood. Studies have shown that the spalted fungi are preferring alder, maple, poplar, phoebe, birch and beech (Chapela 1994; Robinson et al. 2011; Hai-Shan et al. 2013).

Due to the increase in value associated with spalted wood, efforts had recently been undertaken to utilize the process on low value woods. Of particular interest in southeastern China is the potential for PT (Paulownia) and CL (Cunninghamia lanceolate). Both species are native to China, although they are also commonly cultivated in other ethnic areas. These two species of tree from China has wide range of uses and has fixed value due to the relatively fast growth cycle. But $C L$ is a soft, fine, aromatic, and straight grain wood that is easy to process and suitable for furniture, architecture and other applications. PT has a straight texture, a uniform structure and easy processing. $P T$ is suitable for use in construction, furniture, wood-based panels and other applications. These two species of wood are easy to buy, but are currently underutilized in China, making them ideal for value-added processes.

A substantial body of literature exists on the controlled spalting of hardwoods and their use in woodcraft and furniture. There is currently few known record of controlled spalting being attempted with fast-growing wood. For example, using some of the poorly-formed wood, the wood is subjected to fungal infestation without destroying the board to achieve wood dyeing, and ultimately increase the vassal value of these fast-growing woods.

AP (Arthrinium phaeospermum), VA (Vibrio anguillarum), and $A S$ (Aspergillacea) were used to inoculate two fast-growing wood ( $C L$ and $P T$ ). The purpose of this research was to investigate which unknown spalting fungi might be suitable for inoculation on the fast-growing wood, with the intended result of producing extra pigments that would increase the marketability of the lumber. And screened new spotted fungi and study the ability of fastgrowing wood in fungal staining. Whether fungi that produce large amounts of extracellular pigments can also treat cork under controlled conditions. Successful pigmentation of the fast-growing wood by any of the tested fungi would offer an opportunity for sawmills to generate additional income, and then market lumber to interested consumers.

\section{Materials and methods}

\section{Materials}

$P T$ and $C L$ were selected to improve the economic value of fast-growing wood and explore the possibility of producing spalted in fast-growing wood, and the average moisture contents of the tested wood species were $19.04 \%$ for $C L$ and $18.00 \%$ for $P T$, respectively. The specifications of the experimental block were $20 \mathrm{~mm} \times 20 \mathrm{~mm} \times 20 \mathrm{~mm}$.

We recorded whether the pigments of the three fungi changed significantly on both woods. These three wood fungus, which were isolated and screened from spalted $P T$. The strains fungi were shown in Table 1.

\section{Fungi isolation and screening and culturing}

The portion with the spalted was cut into small pieces of length and width less than $10 \mathrm{~mm}$. Alcohol lamps, ultrapure

Table 1 Wood and fungus

\begin{tabular}{lll}
\hline Wood species & Fungi species & $\begin{array}{l}\text { Col- } \\
\text { lection } \\
\text { code }\end{array}$ \\
\hline Cunninghamia lanceolata & Arthrinium phaeospermum & $A P$ \\
& Vibrio anguillarum & $V A$ \\
& Aspergillacea & $A S$ \\
& Arthrinium phaeospermum & $A P$ \\
& Vibrio anguillarum & $V A$ \\
& Aspergillacea & $A S$ \\
\hline
\end{tabular}


water, $0.001 \mathrm{~g} / \mathrm{ml}$ mercury chloride solution, scalpels etc., that were placed in a clean bench and sterilized by UV lamp for $1 \mathrm{~h}$. The small pieces were washed by three times with $0.1 \%$ mercury chloride solution, soaked for 1 min each time, then washed with sterile water for 3-5 min, and finally the wooden blocks were cut with a sterile scalpel on a sterile workbench. The block was placed on the surface of the medium, and the sealed medium was cultured in a dark environment of $27{ }^{\circ} \mathrm{C} \pm 2{ }^{\circ} \mathrm{C}$ for about one week. After the obvious mycelium grows around the small wooden block in the medium, the purification was repeated by $2-3$ times according to different morphological colors until obtaining a purer fungus.

\section{Inoculation}

The moisture content test was performed using a modified decay jar test with vermiculite instead of soil, as outlined in Robinson et al. (2009), to avoid eventual influence of soil substrates on pigment formation. Jars with plastic lids $(250 \mathrm{~mL})$ were prepared with $15 \mathrm{~g}$ vermiculite and $30 \mathrm{~mL}$ water. Before incubating the fungi, blocks were oven dried at $60{ }^{\circ} \mathrm{C}$ for $48 \mathrm{~h}$ and weighed, and then a piece of wood was put into the jar.

Culture jars with vermiculite, water and wood samples were autoclaved for $40 \mathrm{~min}$ at $121{ }^{\circ} \mathrm{C}$. The entire sterile bench was UV sterilized for $30 \mathrm{~min}$ before inoculated. After sterilizing, the inoculum strip (approximately $20 \mathrm{~mm} \times 20 \mathrm{~mm}$ agar with actively growing mycelium) was placed on both sides of the block (longitudinal section), and the block was covered with perlite, and the jar was sealed. Inoculum consisted of mycelium and agar approximately $20 \mathrm{~mm} \times 20 \mathrm{~mm}$ cut from an actively growing culture in a Petri dish. Each fungus was repeated in 20 jars for one wood, and a total of 120 jars were used. After inoculating, jars were placed in an incubation room $\left(27^{\circ} \mathrm{C} \pm 2{ }^{\circ} \mathrm{C}, 80 \% \pm 5 \%\right.$ $\mathrm{RH})$ and incubated in the dark. There were 20 replicate jars of each pairing made (Table 1). After meeting the incubation time, mycelium was scraped off and blocks were evaluated externally for bleaching, pigmentation, and zone lines. Blocks were then cut in half perpendicular to the grain on the transverse plane and one internal side was also evaluated. All internal spalting, pigmentation, and zone lines were noted for the internal face being evaluated. At 4, 6, 8, and 10 weeks, five jars of each combination (a fungus combines a wood) were removed, and those blocks were cleaned, oven dried $\left(60{ }^{\circ} \mathrm{C}\right.$ for $\left.48 \mathrm{~h}\right)$, evaluated for color change, and then weighed again (to determine percent weight loss).

\section{Pigment evaluation}

Pigment evaluation involved RGB distribution, amount of spalting and dyeing depth. Firstly, the wood samples inoculated fungi were dried and scanned with Epson Expression 10000XL Scanner at 2400 dpi to obtain stain images, by which external spalting evaluation were carried out with Scion Image software, following the protocol described in Robinson et al. (2009). Secondly, RGB of these images were analyzed to explore color change three fungal-forming spalted wood by MATLAB. Finally, the sample after being scanned was cut in half to expose the inner plane and the inner surface was also scanned to analyze the internal staining. Meanwhile, the depth of internal spalted was measured by vernier caliper. And five points were selected on both sides and the average value was taken as the dyeing depth of the wood.

\section{Weight loss analysis}

Before inoculating the fungus in the wood block, the wood block was dried for $48 \mathrm{~h}$, and the $M$ was weighed; after completing the culture, the surface impurities of the wood block were taken out, dried, and weighed $m_{0}$. Where, $\mathrm{M}$ is oven dry weight of wood sample prior to exposure and $m_{0}$ is the oven dry weight following exposure to fungus. The calculation for weight loss rate $(R)$ is as Eq. 1:

$R=\frac{M-m_{0}}{M} \times 100 \%$

\section{Results and discussion}

\section{Multivariate analysis}

Multivariate analysis was performed by using fungi, wood species, and culture time as independent variables to determine which fungus produced the greatest amount of spalting in the experiment, and which wood was more suitable for spalted fungal inoculation and optimal incubation time.

\section{RGB distribution}

The obtained RGB values (Tables 2 and 3 ) were compared with the RGB color query comparison table to obtain their expression color. Three fungi were inoculated on $C L$, and the color changes after different incubation time were shown in Fig. 1.

The three columns of Fig. 1 were the average RGB color block of the corresponding $C L$ block at week $4,6,8$, and 10 , inoculated by $A P, V A$ and $A S$. And the specific code of corresponding color blocks (RGB color comparison table) of each color were shown on the right side of the column. From Fig. 1, we could find that when $A P$ was inoculated on $C L$, the color of the wood changed from orange to dark red as the 
Table 2 RGB value of $C L$ (Cunninghamia lanceolate)

\begin{tabular}{|c|c|c|c|c|c|c|c|c|c|c|c|c|}
\hline \multirow[t]{3}{*}{ Fungus } & \multicolumn{12}{|c|}{ Week } \\
\hline & \multicolumn{3}{|l|}{4} & \multicolumn{3}{|l|}{6} & \multicolumn{3}{|l|}{8} & \multicolumn{3}{|l|}{10} \\
\hline & $\mathrm{R}$ & $\mathrm{G}$ & B & $\mathrm{R}$ & $\mathrm{G}$ & B & $\mathrm{R}$ & $\mathrm{G}$ & B & $\mathrm{R}$ & $\mathrm{G}$ & B \\
\hline$A P$ & 200 & 134 & 85 & 203 & 108 & 55 & 152 & 85 & 49 & 147 & 79 & 54 \\
\hline$V A$ & 218 & 170 & 104 & 227 & 172 & 93 & 177 & 147 & 105 & 167 & 134 & 92 \\
\hline$A S$ & 215 & 165 & 103 & 227 & 164 & 93 & 165 & 134 & 94 & 162 & 131 & 92 \\
\hline
\end{tabular}

\begin{tabular}{|c|c|c|c|c|c|c|c|c|c|c|c|c|}
\hline \multirow[t]{3}{*}{ Fungus } & \multicolumn{12}{|c|}{ Week } \\
\hline & \multicolumn{3}{|l|}{4} & \multicolumn{3}{|l|}{6} & \multicolumn{3}{|l|}{8} & \multicolumn{3}{|l|}{10} \\
\hline & $\mathrm{R}$ & $\mathrm{G}$ & B & $\mathrm{R}$ & $\mathrm{G}$ & B & $\mathrm{R}$ & $\mathrm{G}$ & B & $\mathrm{R}$ & $\mathrm{G}$ & B \\
\hline$A P$ & 239 & 208 & 168 & 228 & 193 & 144 & 133 & 124 & 112 & 194 & 185 & 166 \\
\hline$V A$ & 240 & 219 & 162 & 245 & 224 & 159 & 203 & 202 & 177 & 206 & 199 & 165 \\
\hline$A S$ & 240 & 227 & 182 & 246 & 230 & 175 & 205 & 199 & 189 & 201 & 203 & 185 \\
\hline
\end{tabular}

Table 3 RGB value of $P T$ (Paulownia)

\begin{tabular}{|c|c|c|c|c|c|c|}
\hline Time & Color & Color value & Color & Color value & Color & Color value \\
\hline At week 4 & & c98655 & & d7a568 & & d6aa68 \\
\hline At week 6 & & $\operatorname{cb} 6 c 37$ & & e3a55d & & e4ad5d \\
\hline At week 8 & & 985631 & & a5865e & & b19469 \\
\hline At week 10 & & $944 f 37$ & & $\mathrm{a} 2845 \mathrm{~d}$ & & a7875d \\
\hline
\end{tabular}

Fig. 1 Color expressed by RGB value of $C L$ (Cunninghamia lanceolate)

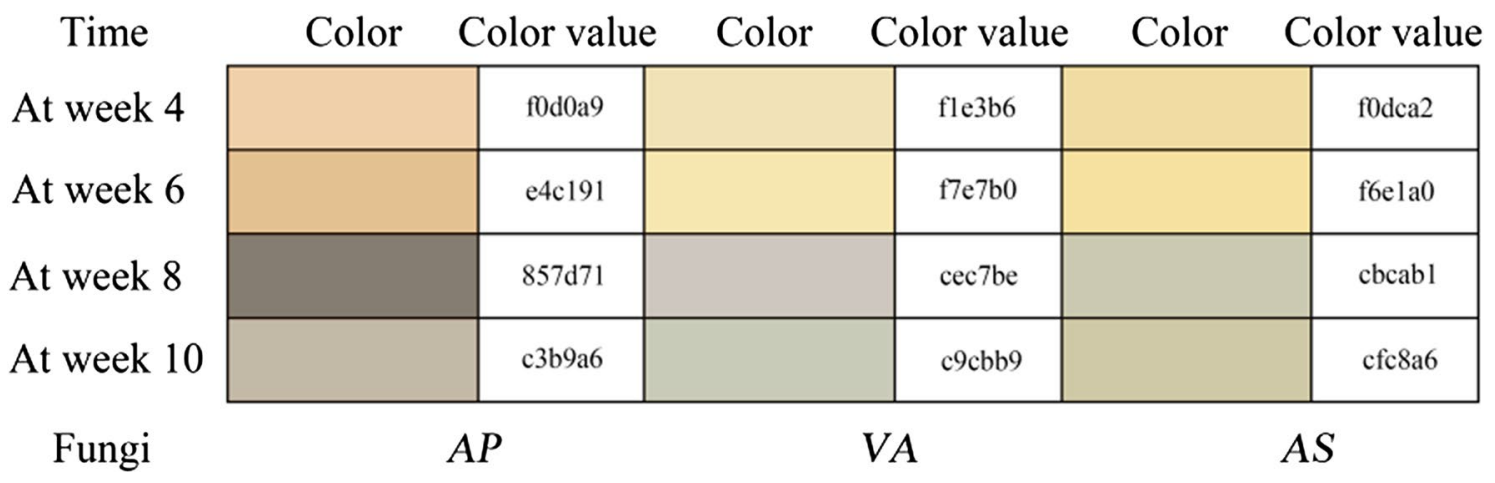

Fig. 2 Color expressed by RGB value of PT (Paulownia)

incubation time prolonged, but from light yellow to taupe for $V A$ and $A S$. Moreover, during the first three incubation times, the color changed from orange to red; and the longer the incubation time was, the deeper the wood color was. During the last period, there was almost not color change except $A P$. The main reason was the pigment secreted by the fungus. It also could be seen that the third inoculated period, that is week 8 , was the best time for dyeing $C L$ for the three fungi, which agreed with the result of Robinson et al. (2007a, b).

From Fig. 1, it could be found that the maximum change of RGB was R, followed by B and G. With the extension of incubation time, the $R$ and $G$ values of wood decreased significantly for the three fungi. But the $\mathrm{B}$ value was influenced significantly by $A P$, and there is no influence for the other 
two fungi. This indicated that the wood color change was more biased towards red and green gray scales.

Figure 2 showed the color change of $P T$. With the extension of the incubation period, the $\mathrm{R}$ and $\mathrm{G}$ values of $P T$ have been significantly reduced. On $P T, A P$ caused the most obvious change in wood color (The largest decrease at week 8), while $V A$ and $A S$ plants had not significant change. When $V A$ and $A S$ were inoculated into the $P T$, the $\mathrm{B}$ value was increased slightly at week 4, 6 and 8, but it almost did not change at week 10 . On the contrary, $A P$ was a special case, the $B$ value was decreased significantly, and rebounded significantly at week 10 , the color approached wood of the firstly incubation time. On account of, $A P$ produced white rot at week 10, which causes wood color to gray.

From the Fig. 2 we could find that when $A P$ was inoculated into $P T$, the color of the wood gradually changed from light yellow to gray as the incubation time prolonged, and eventually became grayish white; then for $V A$, the color of the wood gradually changed from light yellow to gray as the culture period prolonged, and eventually became pale grayish green at week 10; finally for $A S$, the color of the wood gradually changed from light yellow to gray as the incubation time was extended, and finally became pale grayish brown in the fourth incubation time. From this, we knew that $V A$ and $A S$ were not produce effective pigments to cause pronounced color difference in the wood. It could be seen that the color change of wood was not obvious at week 4 with 6 , but significant changes occurred at week 8 . At week 10 , there were significantly external white rot on wood blocks of inoculated with $A P$, but other two fungi were not produced. This suggests that $A P$ might be a white rot fungus.

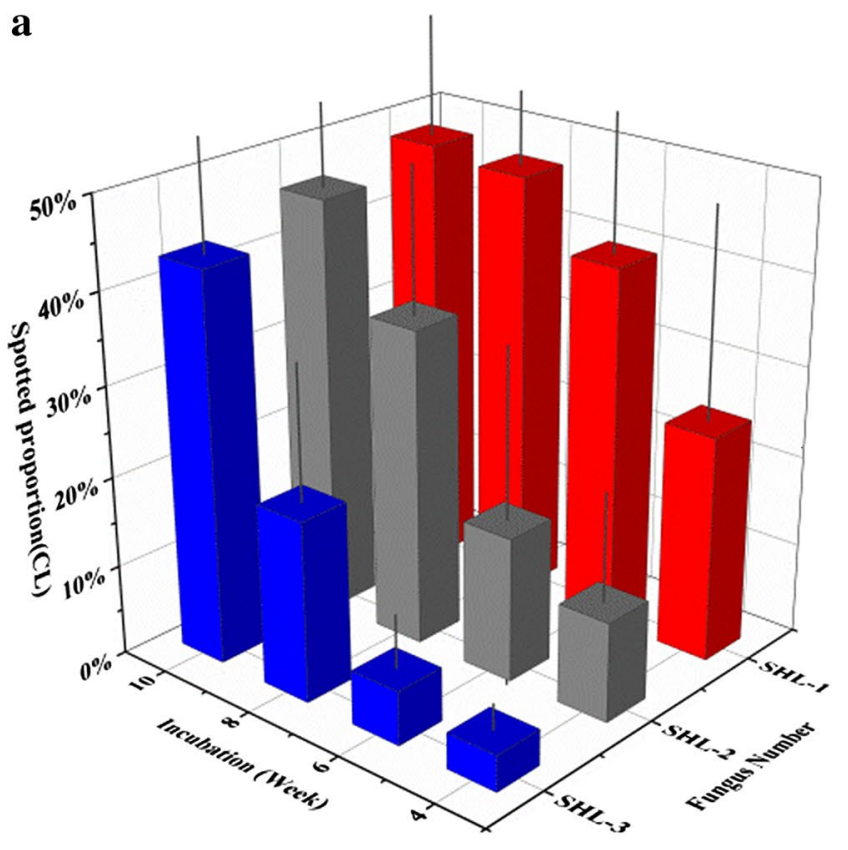

In conclusion, on the one hand, $A P$ could produce obvious red matter on $C L$ and $P T$, among them the staining effect was not stable on $P T$, but $C L$ was perfect. After the sample was placed for one year, the dyeing effect still existed on $C L$. It indicated the potential of $A P$ for large-scale dyeing of wood. On the other hand, the incubation time has a close relationship with the color change of wood. The longer the incubation time, the more the amount of spalting produced by fungus, resulted in a more pronounced change in the color of the wood. The third incubation time might be the best period of pigmented material produced by the fungus during this period (Not sure the most pigmented period).

\section{Amount of spalting}

Figure 3 was the percentage average of external spalting of the wood formed by the three fungi on $C L$ (Fig. 3a) and $P T$ (Fig. 3b). From Fig. 3a, there was significantly more spalting on $C L$ at week 10 than those at week 4,6 and $8(P<0.05)$. And the maximum spalting percentage was $48 \%$ for $A P, 46 \%$ for $V A$, and $43 \%$ for $A S$ at week 10 , and the minimum spalting was $4 \%, 11 \%$ and $25 \%$, respectively, for $A P, V A$ and $A S$ at week 4 . With the extension of inoculated time, the percent of spalting gradually increased. For $C L$, there was more spalting for $A P$ than those of the two other fungi. From Fig. 3b, the maximum spalting on $P T$ was at week 10 for $V A$ and $A S$, but at week 8 for $A P$. The highest value was significant by SPSS one-way ANOVA $(P<0.05)$. The spalting caused by $A P$ and $A S$ was climbed steadily at week $4,6,8$ and 10 , and there was similar trend for $V A$ expect at week 10, at which it was dropped down. It might be an unexpected result of the experiment.

b

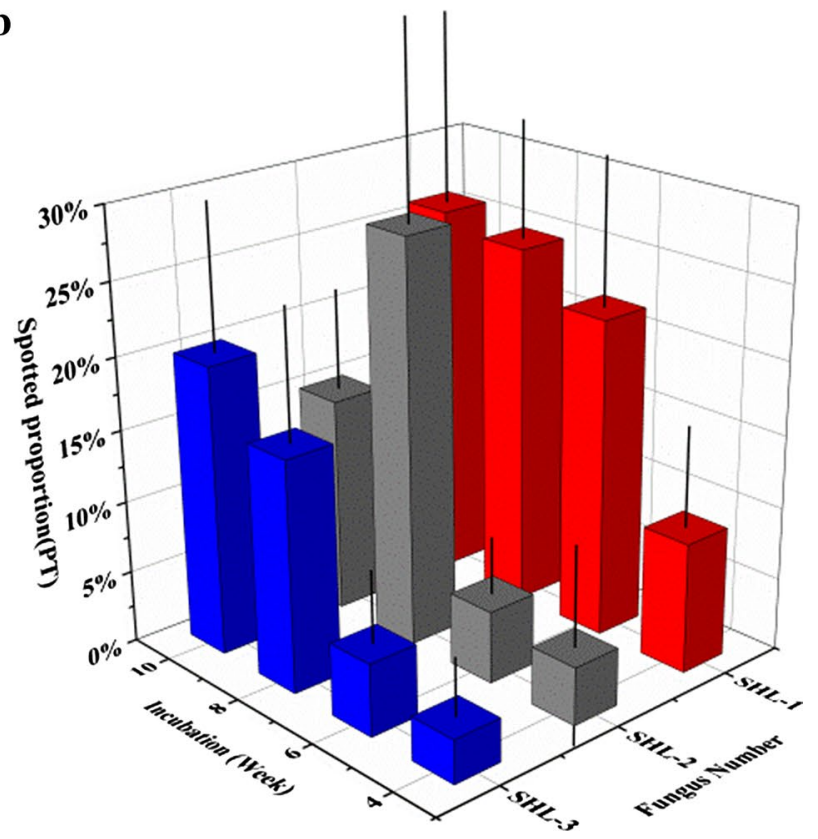

Fig. 3 a The percentage of spalting on $C L$; bThe percentage of spalting on $P T$ 
Fig. 4 a The part effect of three fungus dyeing on $C L$; $\mathbf{b}$ The part effect of three fungus dyeing on $P T$ a

Fungi

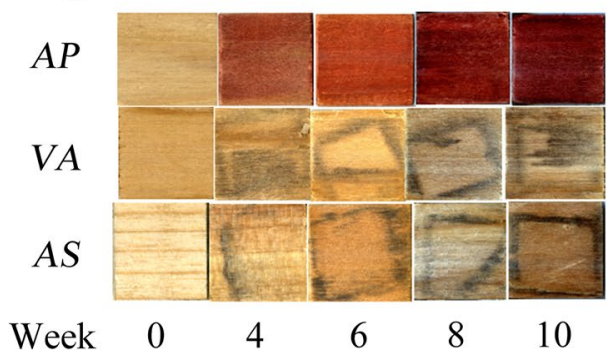

b

Fungi

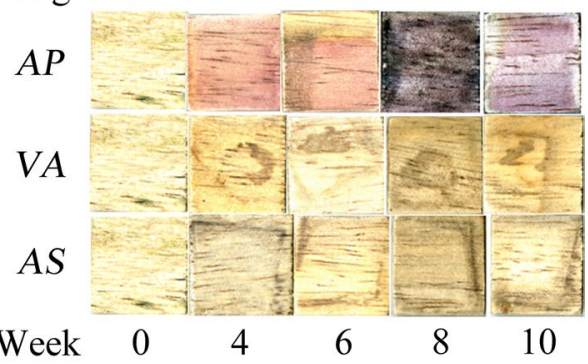

Comparison of three fungus inoculated in $C L$ and $P T$ (Fig. 4). The external spalting on the $C L$ were more than that in the $P T$ at same incubation time and fungus $(P<0.05)$. Among the three fungi, $A P$ external spalting was significantly higher than the other fungus at same incubation time and wood species $(P<0.05)$. Fungi had different preferences for wood, for example, $A P$ preferring to $C L$. It was possible that there were some special matter in $C L$, and it could be beneficial to colonization of fungi and promote the growth of fungi or the production of pigment. In addition, $P T$ might had some substances that inhibit the growth of fungi. Therefore, there had more pigment on $C T$ that $P T$.

\section{Dyeing depth analysis}

The combination of $A P$ and $C L$ was the only one that could produce internal spalting. Therefore, the depths of these wooden blocks were measured, and the average of the depths on both sides of the spalted wood block was taken as the dyeing depth of the wood block. The average value of five test samples per batch was taken as the dyeing depth, and the curve of dyeing depth and percentage of internal spalting as the incubation time. Figure 5 shows the percentage average of internal spalting at different incubation time, and dyeing depth. The mean number of internally spalting and dyeing depth varied significantly depending on time. Internal spalting and dyeing depth were steadily increased for weeks 4, 6, 8 and 10. Later weeks ( 8 and 10) showed significantly more internally spalting than earlier weeks. It could be seen from Fig. 5 that the minimum average depth was $1.8 \mathrm{~mm}$ at week 4, and the maximum average depth was $5.06 \mathrm{~mm}$ at week 10 . In addition, the dyeing depth of a single wood block reached $7 \mathrm{~mm}$ at week 10. Summarizing the internal spalting of $A P$, it could be found that the extension of the incubation time has a significant influence and regularity on the generation of internal spalting.

In the early stages, $A P$ could observe significant pigment diffusion on the solid medium, so the pigment might be an extracellular pigment with good diffusibility. So, as the incubation time, the more pigments were produced by the fungus, the deeper the pigment penetrates the wood and

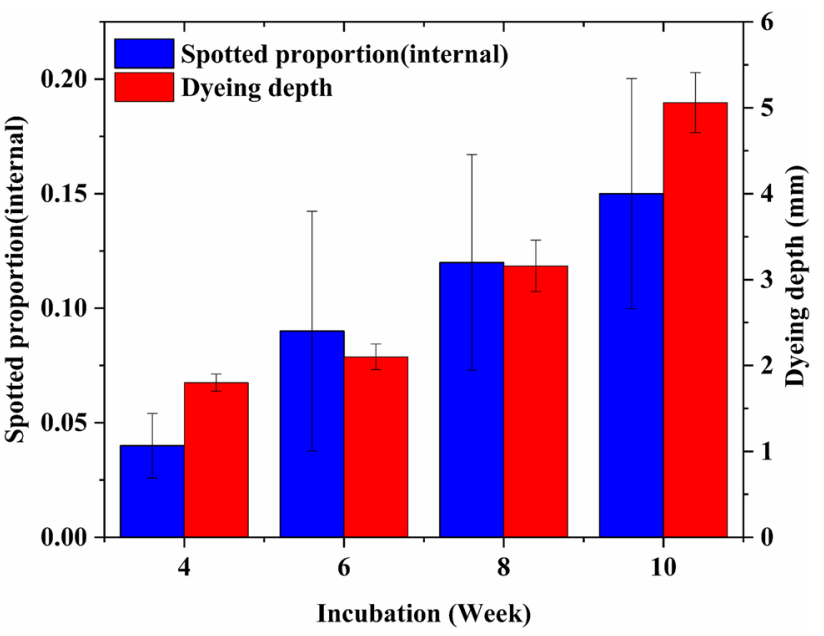

Fig. 5 The depth of internal spalting formed by fungi $A P$ on $C L$ blocks, and the percentage of internal spalting by fungi $A P$ on $C L$ blocks

the larger the proportion of internal spalting. However, it was not prove that the depth of the pigment was caused by physical infiltration or colonization of hyphae in the experiment. Again, results indicate that $A P$ was a better fungus for spalting due to the higher levels of dyeing depth and internally spalting.

\section{Weight loss}

Figure 6 showed the weight loss of the spalted wood block, when the three fungi were inoculated on two fast-growing wood.

Significant difference was analyzed by SPSS one-way ANOVA, and it was found that the influence of incubation time on wood weight loss was not significant $(P>0.05)$. When the three fungus were inoculated into $P T$, the maximum weight loss rate was $16.27 \%$ ( $A P$, at week 10$)$, and the minimum value was $0.40 \%$ ( $A S$, at week 4$)$. On $C L$, the maximum weight loss rate was $1.92 \%$ ( $A P$, at week 6$)$, and the minimum value was $0.90 \%(A S$, at week 4$)$. When $A P$ was inoculated, the weight loss of $P T$ and $C L$ was significantly 


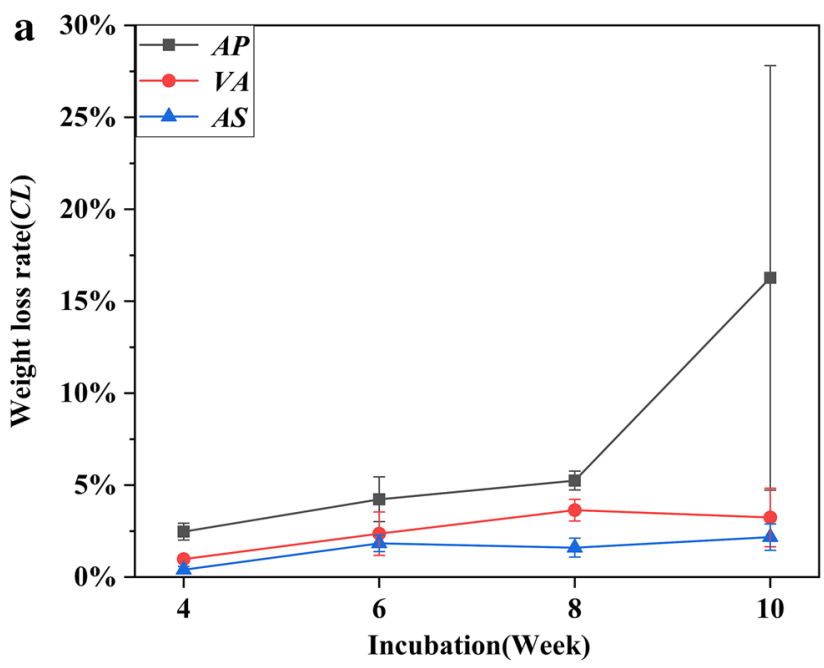

Fig. 6 a Weight loss rate of $C L$ block; b) Weight loss rate of $P T$ block

different. Surprisingly, $P T$ had lower external spalting (pigment), but significantly higher weight loss than $C L$. Especially, the weight loss of $P T$ was significantly higher than that of $C L$ at week 10, and the other two fungi were not exception. Among the three fungi, only $P T$ inoculated with $A P$ showed a regular increase in weight loss with the extension of incubation time, but other fungus and wood combination was not obvious change. Combined with the weight loss rates of the wood, the results showed that there was no significant relationship between the weight loss of $C L$ blocks with fungi and incubation time.

\section{Conclusion}

In this work, $A P$ (Arthrinium phaeospermum), VA (Vibrio anguillarum) and $A S$ (Aspergillacea) were inoculated on $C L$ (Cunninghamia lanceolate) and PT (Paulownia), respectively, and then wood samples were evaluated. The results showed that $A P$ could produce the most percentage average of external and internal spalting on $C L$, which were $48 \%$ and $15 \%$, respectively (The average staining depth was $5.06 \mathrm{~mm}$ ). And the weight loss of the combination was low $(0.46 \mathrm{~g})$, which had little influence on wood strength. The results showed that $A P$ was an ideal wood staining fungus, which could produce pigment stably and had a broad application prospect. $C L$ was an ideal application in wood fungus dyeing.

Open Access This article is licensed under a Creative Commons Attribution 4.0 International License, which permits use, sharing, adaptation, distribution and reproduction in any medium or format, as long as you give appropriate credit to the original author(s) and the source,

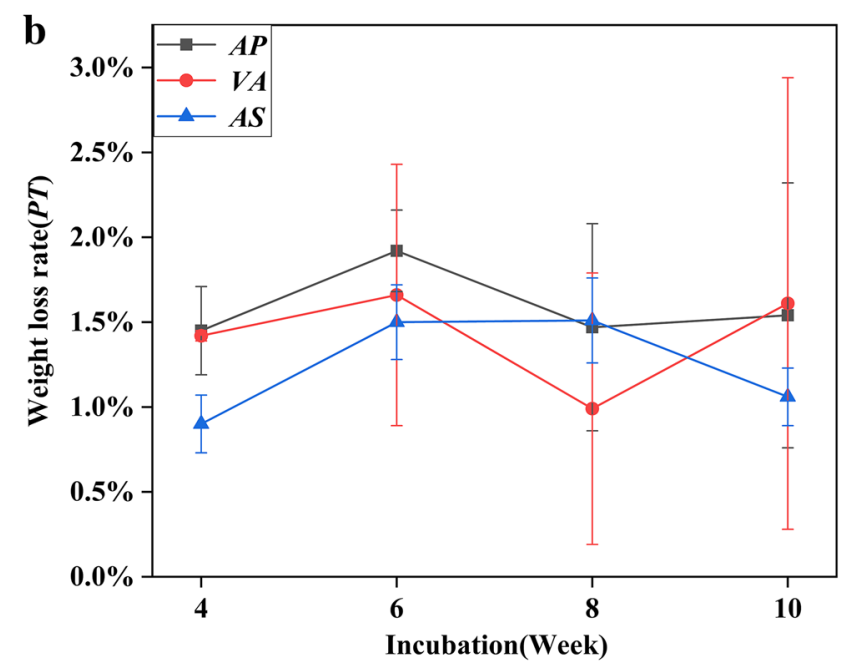

provide a link to the Creative Commons licence, and indicate if changes were made. The images or other third party material in this article are included in the article's Creative Commons licence, unless indicated otherwise in a credit line to the material. If material is not included in the article's Creative Commons licence and your intended use is not permitted by statutory regulation or exceeds the permitted use, you will need to obtain permission directly from the copyright holder. To view a copy of this licence, visit http://creativecommons.org/licenses/by/4.0/.

\section{References}

Agurto MEP, Gutierrez SMV, Chen HL, Robinson SC (2017) Woodrotting fungal pigments as colorant coatings on oil-based textile dyes. Coatings 7(152):152. https://doi.org/10.3390/coatings71 00152

Blanchette RA, Biggs AR (1992) Defense mechanisms of woody plants against fungi. Springer, Berlin. ISBN: 978-3-662-01642-8

Cease KR, Blanchette RA, Highley TL (1989) Interactions between Scytalidium species and brown- or white-rot basidiomycetes in birch wood. Wood Sci Technol 23(2):151-161

Chapela IH (1994) Spalted beech wood. Mycologist 8(4):186-186

Hai-Shan HE, Qiu J, Gan CY (2013) Research status and prospect on spalted wood. J Southwest For Univ 33(6): 94-98 (Chinese with English abstract)

Hinsch EM, Robinson SC (2018) Comparing colorfastness to light of wood-staining fungal pigments and commercial dyes: an alternative light test method for color fastness. Coatings 8(5):189. https ://doi.org/10.3390/coatings8050189

Hinsch EM, Weber G, Chen HL, Robinson SC (2015) Colorfastness of extracted wood-staining fungal pigments on fabrics: A new potential for textile dyes. J Text Apparel Technol Manag 9(3):1-11

Liese W (1970) Ultrastructural aspects of woody tissue disintegration. Annu Rev Phytopathol 8(8):231-258

Mallett KI, Hiratsuka Y (1986) Nature of the "black line" produced between different biological species of the Armillaria mellea complex. Can J Bot 64(11):2588-2590. https://doi.org/10.1139/ b86-342 
Richter DL, Glaeser JA (2015) Wood decay by Chlorociboria aeruginascens (Nyl.) Kanouse (Helotiales, Leotiaceae) and associated basidiomycete fungi. Int Biodeterior Biodegrad 105:239-244

Robinson SC (2012) Developing fungal pigments for "painting" vascular plants. Appl Microbiol Biotechnol 93(4):1389-1394

Robinson SC, Laks PE (2010a) The effects of copper in large-scale single-fungus and dual-fungi wood systems. For Prod J 60(6): 490-495. https://doi.org/10.13073/0015-7473-60.6.490

Robinson SC, Laks PE (2010b) Wood species affects laboratory colonization rates of Chlorociboria sp. Int Biodeterior Biodegrad 64(4):305-308. https://doi.org/10.1016/j.ibiod.2010.03.003

Robinson SC, Laks PE (2010c) Wood species and culture age affect zone line production of Xylaria polymorpha. Open Mycol J 4(1):18-21. https://doi.org/10.2174/1874437001004010018

Robinson SC, Cooper PA (2011) Wood preference of spalting fungi in urban hardwood species. Int Biodeterior Biodegrad 65(8):11451149. https://doi.org/10.1016/j.ibiod.2011.07.012

Robinson SC, Richter DL, Laks PE (2007a) Colonization of sugar maple by spalting fungi. For Prod J 57(4):24-32

Robinson SC, Laks PE, Richter DL, Pickens JB (2007b) Evaluating loss of machinability in spalted sugar maple. For Prod J 57(4):33-37

Robinson SC, Laks PE, Turnquist EJ (2009) A method for digital color analysis of spalted wood using scion image software. Materials 2(1):62-75. https://doi.org/10.3390/ma2010062

Robinson SC, Hinsch E, Weber G, Freitas S (2014a) Method of extraction and resolubilisation of pigments from Chlorociboria aeruginosa and Scytalidium cuboideum, two prolific spalting fungi. Color Technol 130(3):221-225. https://doi.org/10.1111/ cote. 12080

Robinson SC, Weber G, Hinsch E, Gutierrez SMV, Pittis L, Freitas S (2014b) Utilizing extracted fungal pigments for wood spalting - a comparison of induced fungal pigmentation to fungal dyeing. $\mathbf{J}$ Coat 2014:1-8. https://doi.org/10.1155/2014/759073

Robinson SC, Gutierrez SMV, Garcia RAC, Iroume N, Vorland NR, Mcclelland A, Huber M, Stanton S (2017) Potential for carrying dyes derived from spalting fungi in natural oils. J Coat Technol Res 3(1): 1-7. https://doi.org/10.1007/s11998-017-9919-4

Vega Gutierrez SM (2016) Spalting fungi: genetic identification, material interactions and microscopical characteristics of extracted pigments. Oregon State University Press, Oregon, p 70

Weber G, Chen HL, Hinsch E, Freitas S, Robinson S (2015) Pigments extracted from the wood-staining fungi Chlorociboria aeruginosa, Scytalidium cuboideum, and S. ganodermophthorum show potential for use as textile dyes. Coloration Technology 130(6): 445-452. https://doi.org/10.1111/cote.12110

Worrall JJ, Anagnost SE, Zabel RA (1997) Comparison of wood decay among diverse lignicolous fungi. Mycologia 89(2):199-219. https ://doi.org/10.1080/00275514.1997.12026772

Publisher's Note Springer Nature remains neutral with regard to jurisdictional claims in published maps and institutional affiliations. 